\title{
Robust Stability of Fractional Order Time-Delay Control Systems: A Graphical Approach
}

\author{
Radek Matušů and Roman Prokop \\ Centre for Security, Information and Advanced Technologies (CEBIA-Tech), Faculty of Applied Informatics, \\ Tomas Bata University in Zlín, nám. T. G. Masaryka 5555, 76001 Zlín, Czech Republic
}

Correspondence should be addressed to Radek Matušů; rmatusu@fai.utb.cz

Received 16 March 2015; Accepted 14 June 2015

Academic Editor: Haranath Kar

Copyright (C) 2015 R. Matušů and R. Prokop. This is an open access article distributed under the Creative Commons Attribution License, which permits unrestricted use, distribution, and reproduction in any medium, provided the original work is properly cited.

\begin{abstract}
The paper deals with a graphical approach to investigation of robust stability for a feedback control loop with an uncertain fractional order time-delay plant and integer order or fractional order controller. Robust stability analysis is based on plotting the value sets for a suitable range of frequencies and subsequent verification of the zero exclusion condition fulfillment. The computational examples present the typical shapes of the value sets of a family of closed-loop characteristic quasipolynomials for a fractional order plant with uncertain gain, time constant, or time-delay term, respectively, and also for combined cases. Moreover, the practically oriented example focused on robust stability analysis of main irrigation canal pool controlled by either classical integer order PID or fractional order PI controller is included as well.
\end{abstract}

\section{Introduction}

Recently, the fractional order calculus (FOC) and its engineering applications represent attractive research field with rapidly growing amount of related scientific works. This progress is understandable since the use of differentiation and integration under an arbitrary real or even complex number of the operations provides efficient tool for many real-life problems and since the knowledge of suitable and relatively comprehensible mathematical instruments for fractional order issues has increased lately. The principal sources for studying the FOC are, for example, the monographs [1-3] and possibly also [4] or [5]. The FOC has already been useful in areas such as bioengineering, viscoelasticity, electronics, robotics, control theory, and signal processing $[6,7]$. The examples of several useful control-oriented works can be seen in [8-12]. Obviously, the FOC has influenced also analysis and control of time-delay systems which represent usually complicated but relatively frequent controlled objects [13-17].

Models with parametric uncertainty are popular and effective way to uncertainty modelling and consequently to description of too complicated, nonlinear, or varying reallife systems by means of linear models. In such systems, the structure (model order) is supposed or known, but the parameters are bounded somehow. Typically, they lie within given intervals. One of the related principal tasks consists in robust stability analysis, that is, in investigation of keeping the stability under all possible variations of uncertain parameters. Some authors have already tried to combine the issue of robust stability of systems affected by parametric uncertainty with fractional order systems, for example, [18-28].

This paper is focused on a graphical approach to robust stability analysis and especially on its application to fractional order time-delay control systems. More specifically, the control loop studied in the computational examples consists of a fractional order time-delay plant with uncertain parameters and standard integer order PID controller. The robust stability is tested via plotting the value sets of a closedloop characteristic quasipolynomial and application of the zero exclusion condition. The presented examples include the typical shapes of the value sets for a fractional order controlled system with uncertain gain, time constant, or time-delay term, respectively, and then also for the case of all uncertain parameters together. Moreover, the final processcontrol-oriented example deals with robust stability analysis for main irrigation canal pool controlled by either classical 
PID or fractional order PI controller. This paper is the significantly extended version of the conference contribution [29].

The paper is organized as follows. In Section 2, basic theoretical background and description of fractional order systems are provided. Section 3 then presents the robust stability analysis for integer order and fractional order systems with parametric uncertainty with especial emphasis on the value set concept and the zero exclusion condition. Next, a number of computational examples and visualizations of the value sets for closed loop containing a fractional order time-delay plant with various uncertain parameters are shown in the extensive Section 4. Further, Section 5 contains more specific and practically oriented example motivated by control of main irrigation canals with variable parameters. And finally, Section 6 offers some concluding remarks.

\section{Fractional Order Systems}

The FOC is grounded in generalization of differentiation and integration to an arbitrary (rational, irrational, or even complex) order. This generalization has resulted in the introduction of basic continuous differintegral operator $[1,2,4,6]$ :

$$
{ }_{a} D_{t}^{\alpha}= \begin{cases}\frac{d^{\alpha}}{d t^{\alpha}} & \operatorname{Re} \alpha>0 \\ 1 & \operatorname{Re} \alpha=0 \\ \int_{a}^{t}(d \tau)^{-\alpha} & \operatorname{Re} \alpha<0,\end{cases}
$$

where $\alpha$ is the order of the differintegration (ordinarily $\alpha \epsilon$ $\mathbb{R})$ and $a$ is a constant related to initial conditions. The differintegral can be defined in various ways. The three most common ones are Riemann-Liouville, Grünwald-Letnikov, and Caputo definitions. 9]

The Laplace transform of the differintegral is given by [ 4 ,

$$
\begin{aligned}
L\left\{{ }_{a} D_{t}^{\alpha} f(t)\right\}= & \int_{0}^{\infty} e^{-s t}{ }_{0} D_{t}^{\alpha} f(t) d t \\
= & s^{\alpha} F(s) \\
& -\left.\sum_{m=0}^{n-1} s^{m}(-1)^{j}{ }_{0} D_{t}^{\alpha-m-1} f(t)\right|_{t=0},
\end{aligned}
$$

where integer $n$ lies within $(n-1<\alpha \leq n)$.

The (time-delay-free) fractional order transfer function can be written as $[3,5]$

$$
G(s)=\frac{B\left(s^{\beta_{k}}\right)}{A\left(s^{\alpha_{k}}\right)}=\frac{b_{m} s^{\beta_{m}}+b_{m-1} s^{\beta_{m-1}}+\cdots+b_{0} s^{\beta_{0}}}{a_{n} s^{\alpha_{n}}+a_{n-1} s^{\alpha_{n-1}}+\cdots+a_{0} s^{\alpha_{0}}},
$$

where $a_{k}$ with $(k=0, \ldots, n)$ and $b_{k}$ with $(k=0, \ldots, m)$ denote constants and $\alpha_{k}$ with $(k=0, \ldots, n)$ and $\beta_{k}$ with $(k=0, \ldots, m)$ are arbitrary real numbers. According to $[4,5]$, one can assume inequalities $\alpha_{n}>\alpha_{n-1}>\cdots>\alpha_{0}$ and $\beta_{m}>\beta_{m-1}>\cdots>\beta_{0}$ without loss of generality. In this paper, the controlled time-delay system is supposed generally as

$$
G(s)=\frac{B\left(s^{\beta_{k}}\right)}{A\left(s^{\alpha_{k}}\right)} e^{-\Theta s} .
$$

\section{Robust Stability Analysis under Parametric Uncertainty}

The stability of the closed-loop system will be tested via stability of its characteristic polynomial (or quasipolynomial in the case of this paper).

The continuous-time fractional order uncertain polynomial can have the form

$$
\begin{aligned}
p(s, q)= & \rho_{n}(q) s^{\alpha_{n}}+\rho_{n-1}(q) s^{\alpha_{n-1}}+\cdots+\rho_{1}(q) s^{\alpha_{1}} \\
& +\rho_{0}(q) s^{\alpha_{0}}
\end{aligned}
$$

where $q$ is the vector of uncertainty and $\rho_{k}$ for $k=$ $0,1,2 \ldots, n$ are coefficient functions. Besides, the characteristic quasipolynomial (for closed control loop with time-delay plant) would contain the term $e^{-\Theta s}$.

Then, the family of polynomials is [30]

$$
P=\{p(\cdot, q): q \in Q\},
$$

where $Q$ is the uncertainty bounding set (frequently, it is a multidimensional box).

The family of polynomials (6) is robustly stable if and only if $p(s, q)$ is stable for all $q \in Q$. The choice of technique for investigation of robust stability depends primarily on the structure of uncertainty. Generally, the higher level of relation among coefficients entails more complex robust stability analysis which requires more sophisticated tools. However, one graphical method seems to be unique from the viewpoint of its universality and applicability. It is based on combination of the value set concept and the zero exclusion condition [30]. It can be applied for a wide range of uncertainty structures, from the simplest to the very complicated ones. Moreover, it is applicable also for various regions of stability (robust $D$-stability). The detailed information on parametric uncertainty and robust stability analysis as well as examples of the typical value sets can be found in [30] and subsequently, for example, in [31,32]. And finally, [18-21] have extended the idea of the value set concept also to fractional order uncertain polynomials.

Under assumption of a family of polynomials (6), the value set at frequency $\omega \in \mathbb{R}$ is given by [30]

$$
p(j \omega, Q)=\{p(j \omega, q): q \in Q\} .
$$

It means that $p(j \omega, Q)$ is the image of $Q$ under $p(j \omega, \cdot)$. Practical construction of the value sets can be accomplished by substituting $s$ for $j \omega$, fixing $\omega$, and letting the vector of uncertain parameters $q$ range over the set $Q$.

The zero exclusion condition for Hurwitz stability of family of continuous-time polynomials (6) is defined as follows [30]: assume invariant degree of polynomials in the family, pathwise connected uncertainty bounding set $Q$, continuous coefficient functions $\rho_{k}(q)$ for $k=0,1,2, \ldots, n$, and at least one stable member $p\left(s, q^{0}\right)$. Then the family $P$ is robustly stable if and only if the complex plane origin is excluded from the value set $p(j \omega, Q)$ at all frequencies $\omega \geq 0$; that is, $P$ is robustly stable if and only if

$$
0 \notin p(j \omega, Q) \quad \forall \omega \geq 0 .
$$


Authors of [18-21] construct the value sets of the fractional order families of polynomials mainly on the basis of the fact that the fractional power of $j \omega$ can be written as

$$
(j \omega)^{\alpha}=\omega^{\alpha}\left(\cos \frac{\pi}{2} \alpha+j \sin \frac{\pi}{2} \alpha\right)
$$

and on the consequent analysis of vertices and exposed edges.

In this work, the value sets are plotted for quasipolynomials (closed-loop characteristic quasipolynomials of the feedback circuits with the uncertain time-delay fractional order plant and fixed integer order or fractional order controller) and their visualization is based on sampling the uncertain parameters and on computation of partial points of the value sets for a considered frequency range. Thanks to the applied sampling (brute-force) method, the value sets of quasipolynomials can be easily computed and consequently the robust stability can be investigated with the assistance of standard zero exclusion condition. The technique itself should be clear from the following examples.

\section{Computational Examples: Typical Shapes of Value Sets}

Consider a fractional order time-delay plant given by

$$
G(s, K, T, \Theta)=\frac{K}{T s^{\alpha}+1} e^{-\Theta s}
$$

where $K$ is a gain, $T$ stands for a time constant, $\alpha$ is a real number representing the fractional order of the dynamics, and $\Theta$ is a time-delay term. One or more of the parameters $K, T$, and $\Theta$ are uncertain and they can vary within given intervals.

More specifically, the controlled system is described, for example, as

$$
G(s, K, T, \Theta)=\frac{K}{T s^{0.75}+1} e^{-\Theta s}
$$

where either one of the parameters is uncertain:

$$
\begin{aligned}
& K=[7,13] ; \quad T=3 ; \quad \Theta=2, \\
& K=10 ; \quad T=[2,4] ; \quad \Theta=2, \\
& K=10 ; \quad T=3 ; \quad \Theta=[1.5,2.5]
\end{aligned}
$$

or all of them can lie within supposed bounds:

$$
K=[7,13] ; \quad T=[2,4] ; \quad \Theta=[1.5,2.5] .
$$

In all cases, the nominal system used for the controller design is assumed with the fixed (average) values:

$$
G_{N}(s)=\frac{10}{3 s^{0.75}+1} e^{-2 s}
$$

The PID controller for this plant could be obtained, for example, with the assistance of the FOMCON Toolbox for MATLAB $[33,34]$ and its routine "iopid_tune." More

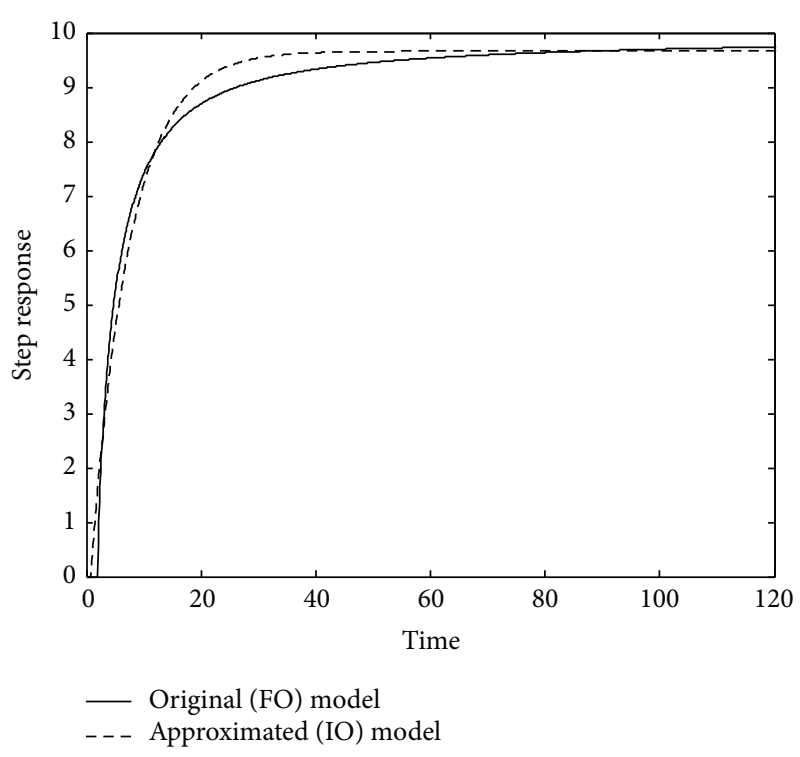

FIGURE 1: Comparison of step responses of original (FO) model (16) and approximated (IO) model (17).

specifically, the Oustaloup filter based [35] approximation leads to the integer order model:

$$
G_{A}(s)=\frac{9.66313}{6.75338 s+1} e^{-0.736803 s} .
$$

The selected controller for this plant has the form

$$
C(s)=K_{p}+\frac{K_{i}}{s}+K_{d} s=0.1+\frac{0.05}{s}+0.01 s .
$$

More information on integer order approximations of fractional order systems can be found, for example, in [36]. The comparison between step responses of the fractional order (FO) model and its integer order (IO) approximation can be seen in Figure 1. It is still obtained through the FOMCON Toolbox.

The control responses for the loops with controller (18) and original nominal (FO) model (16) or approximated (IO) model (17), respectively, are compared in Figure 2.

Nevertheless, the approximation was done only for the sake of IO controller choice. The robust stability of the closedloop control system will be investigated by means of the family of its characteristic quasipolynomials, which contains the true FO model (11):

$$
\begin{aligned}
p_{\mathrm{cl}}(s, K, T, \Theta)= & \left(T s^{0.75}+1\right) s \\
& +K e^{-\Theta s}\left(K_{d} s^{2}+K_{p} s+K_{i}\right),
\end{aligned}
$$

where $K_{p}, K_{i}$, and $K_{d}$ are fixed PID controller parameters from (18) while one or more of the coefficients $K, T$, and $\Theta$ of plant (11) can vary according to (12)-(15).

First, only the gain is supposed to be uncertain while the time constant and time-delay term remain fixed; that is, scenario (12) holds true. 


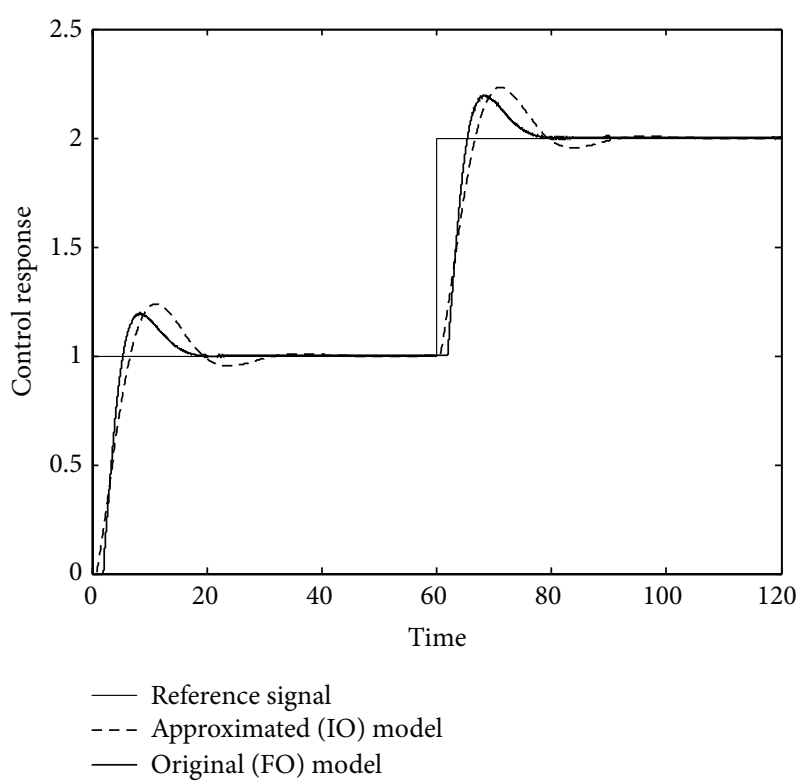

FIGURE 2: Comparison of control responses of original (FO) model (16) and approximated (IO) model (17).

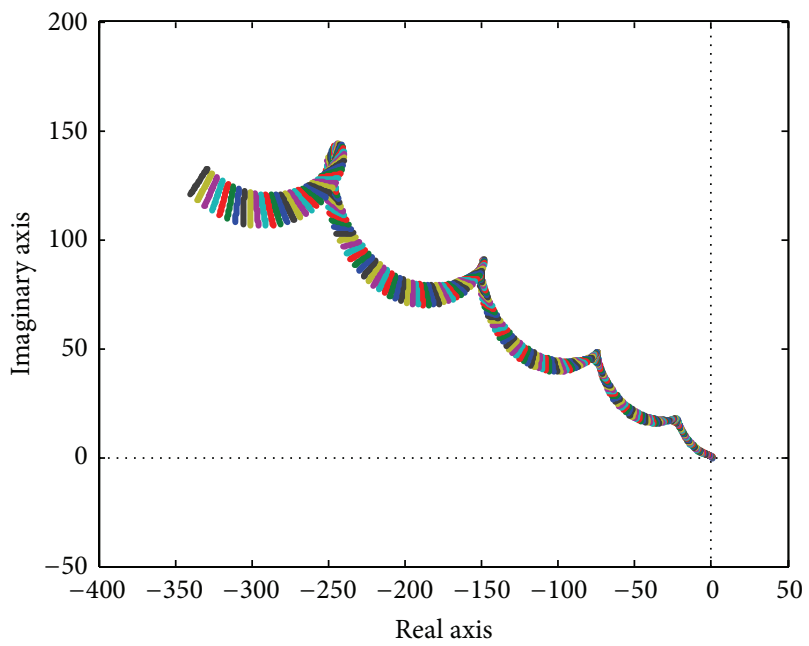

FIGURE 3: Value sets for controller (18) and plant (11) with parameters (12).

The straight-line value sets computed for the corresponding family of closed-loop characteristic quasipolynomials for the range of frequencies from 0 to 15 with the step 0.05 are depicted in Figure 3. At each frequency, $K$ is sampled within given interval with the step 0.1 (i.e., each line consists of 61 points). Then, the zoomed version for better view of the situation near the origin of the complex plane is shown in Figure 4. As can be seen, the zero point is excluded from the value sets. Thus, because the family contains at least one stable member (see Figure 2) and the zero is excluded, the family is robustly stable. In other words, the closed-loop control system with the fractional order uncertain plant (11) with (12) and with fixed PID controller (18) remains stable for all possible values of gain from supposed interval.

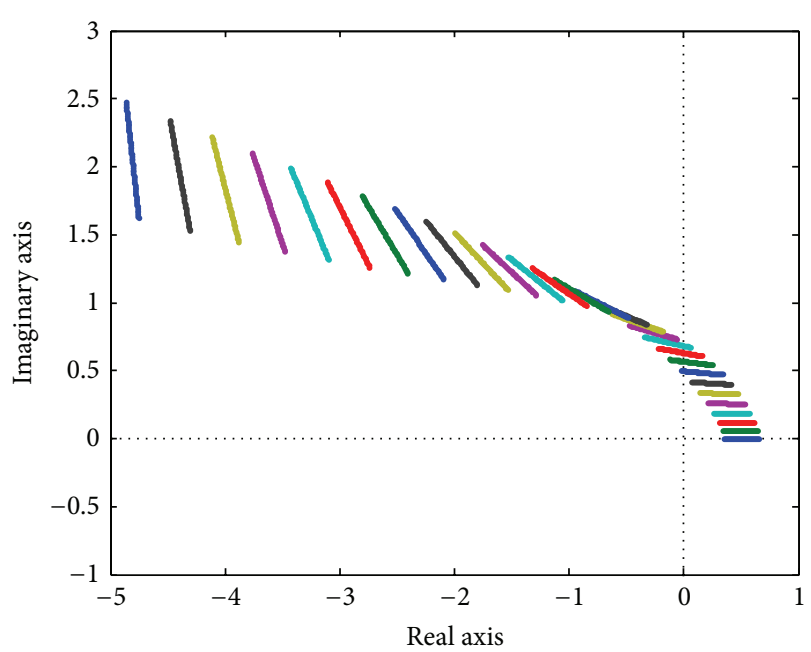

FIGURE 4: Value sets for controller (18) and plant (11) with parameters (12)-zoomed version.

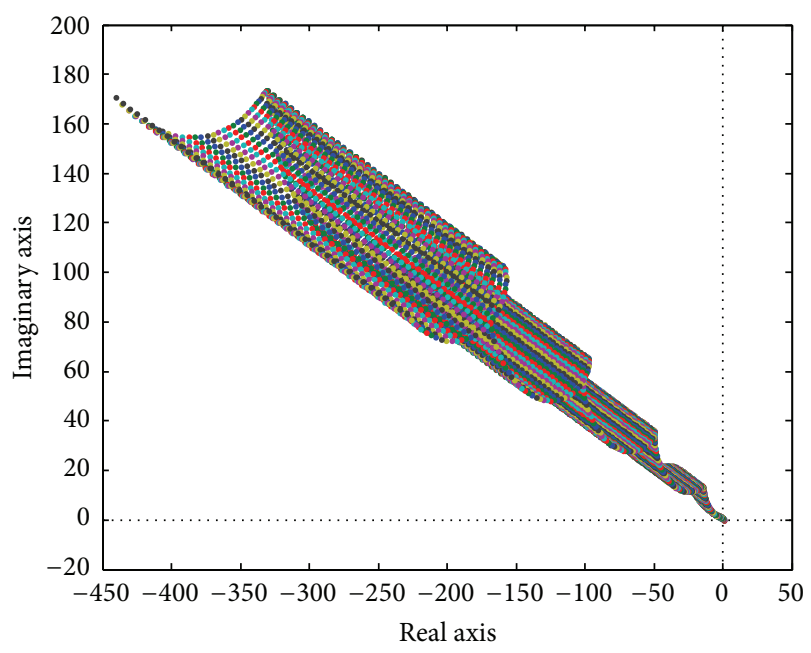

FIGURE 5: Value sets for controller (18) and plant (11) with parameters (13).

Now, the time constant is going to be the only uncertain parameter according to (13). Figure 5 shows the resulting value sets for the family of closed-loop characteristic quasipolynomials (in the same range of frequencies as in the previous case). Again, $T$ is sampled with the step 0.05 and consequently every straight-line value set consists of only 41 points. The closer look to the complex plane origin is provided by Figure 6, which clearly indicates that the zero point is not included in the value sets. Analogically to the previous example, the family can be considered as the robustly stable one.

Next simulation scenario is given by (14); that is, the timedelay term is the uncertain parameter. The respective value sets for again $\omega=0: 0.05: 15$ are shown in Figure 7. Each value set is not a straight-line now but more complex singleparameter curve. Time-delay term is sampled according to $\Theta=1.5: 0.02: 2.5$, which gives the value set as a curve 


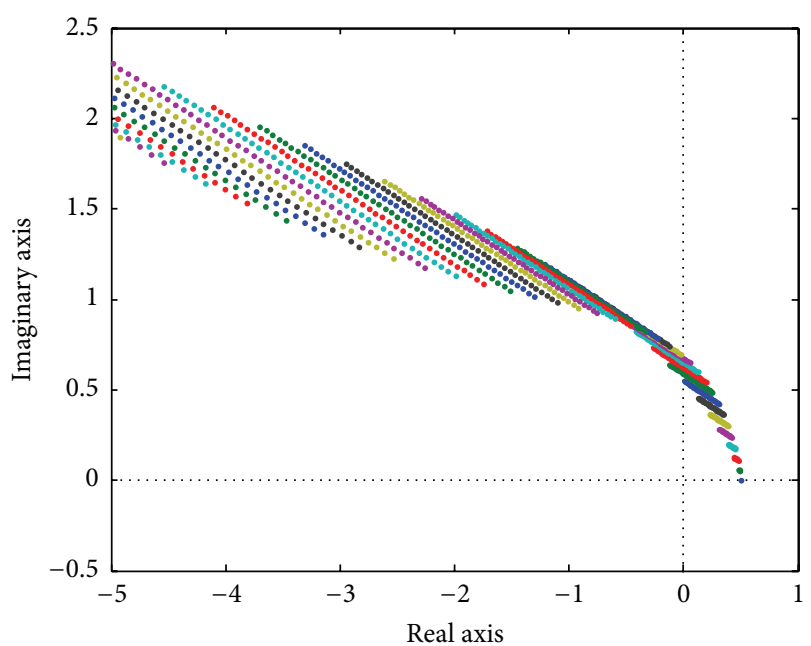

FIGURE 6: Value sets for controller (18) and plant (11) with parameters (13) - zoomed version.

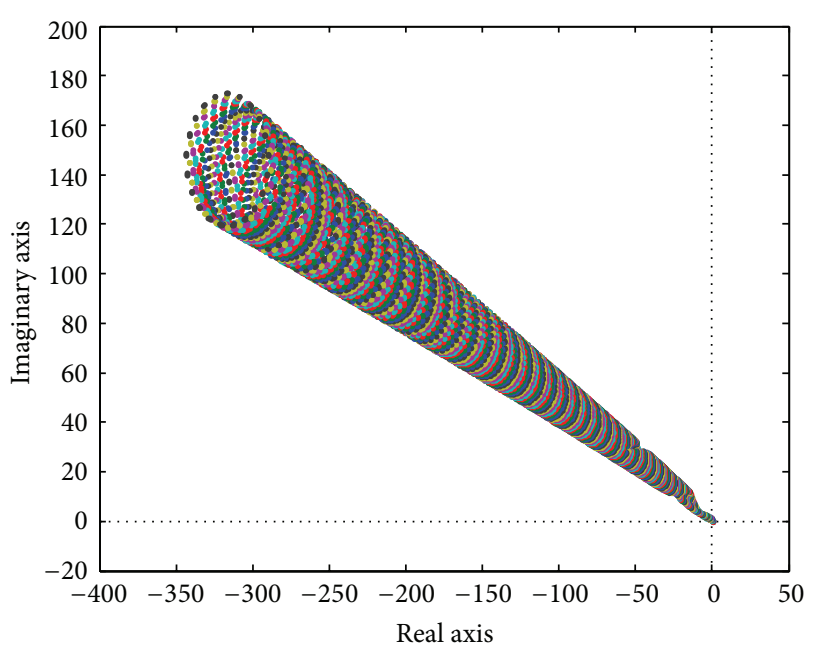

FIGURE 7: Value sets for controller (18) and plant (11) with parameters (14).

plotted via 51 points. The detailed view in Figure 8 reveals that the closed-loop system is robustly stable also in this case.

Finally, the controlled plant with all three varying parameters is assumed-see (15) — and its value sets are plotted in Figure 9. The frequency and the plant parameters are sampled as follows:

$$
\begin{aligned}
& \omega=0: 0.2: 15 \\
& K=7: 0.2: 13 \\
& T=2: 0.1: 4 \\
& \Theta=1.5: 0.04: 2.5 .
\end{aligned}
$$

The family definitely contains a stable member and the zero point is excluded from the value sets (as can be seen from the zoomed Figure 10 where the step of frequency is lowered

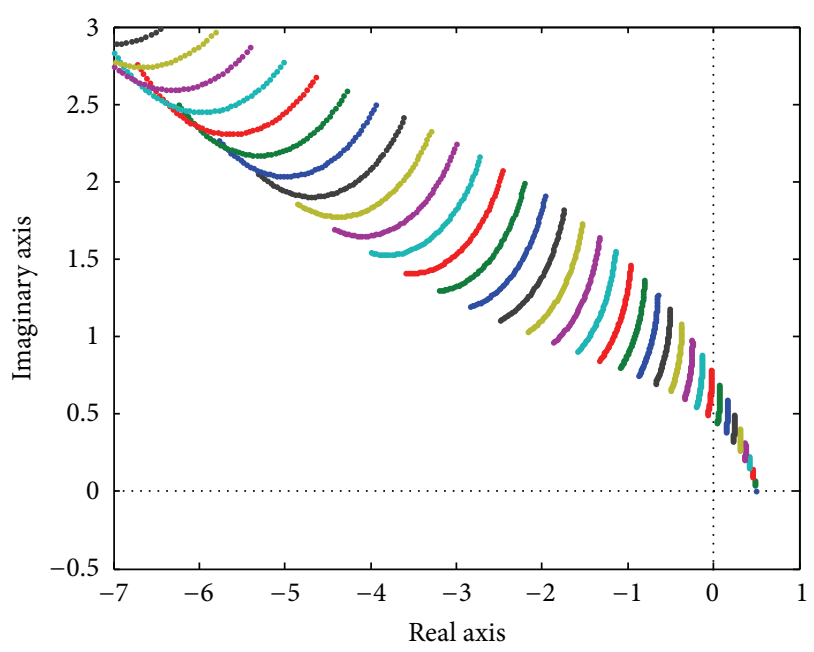

FIGURE 8: Value sets for controller (18) and plant (11) with parameters (14)-zoomed version.

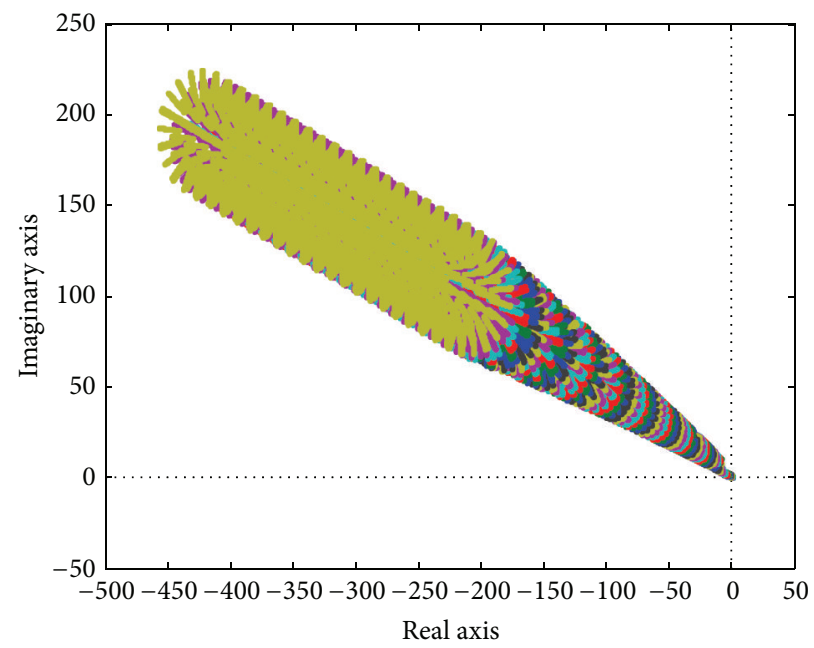

FIGURE 9: Value sets for controller (18) and plant (11) with parameters (15).

to 0.1 ), so the family and the whole closed-loop control system are robustly stable even in this event.

In addition to all robustly stable cases shown in the previous parts, one can very easily obtain the family which is robustly unstable. For example, assume the uncertain gain case (12) with a different PID controller:

$$
C(s)=K_{p}+\frac{K_{i}}{s}+K_{d} s=0.3+\frac{0.15}{s}+0.03 s
$$

which results in the value sets in Figure 11 and its zoomed version in Figure 12 (for $\omega=0: 0.05: 15$ ). Now, the value sets include the complex plane origin and thus the family of closed-loop characteristic quasipolynomials is not robustly stable (i.e., the system would be unstable for some possible values of $K$ ).

Nevertheless, even if the stability test using the zero exclusion condition is visually very simple, one has to be careful 


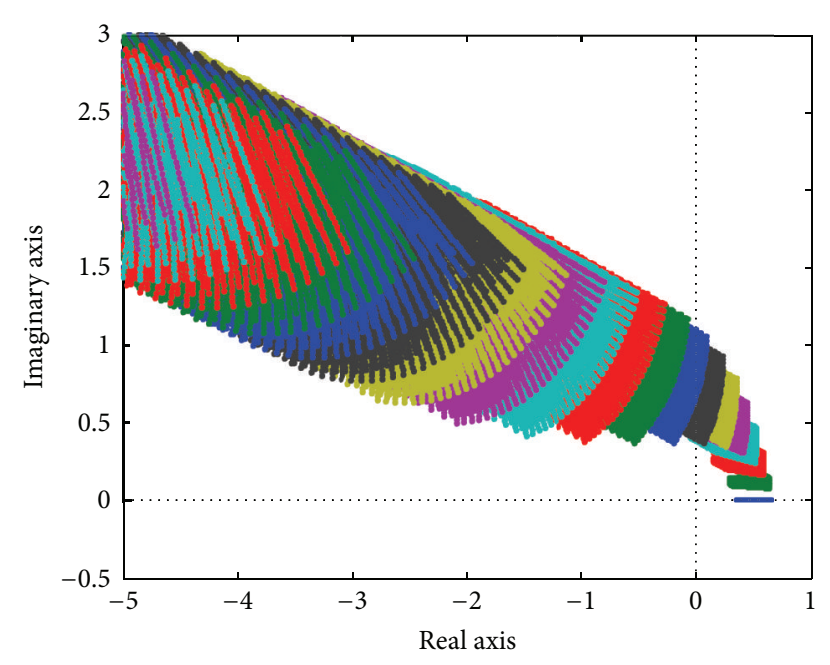

FIGURE 10: Value sets for controller (18) and plant (11) with parameters (15)-zoomed version.

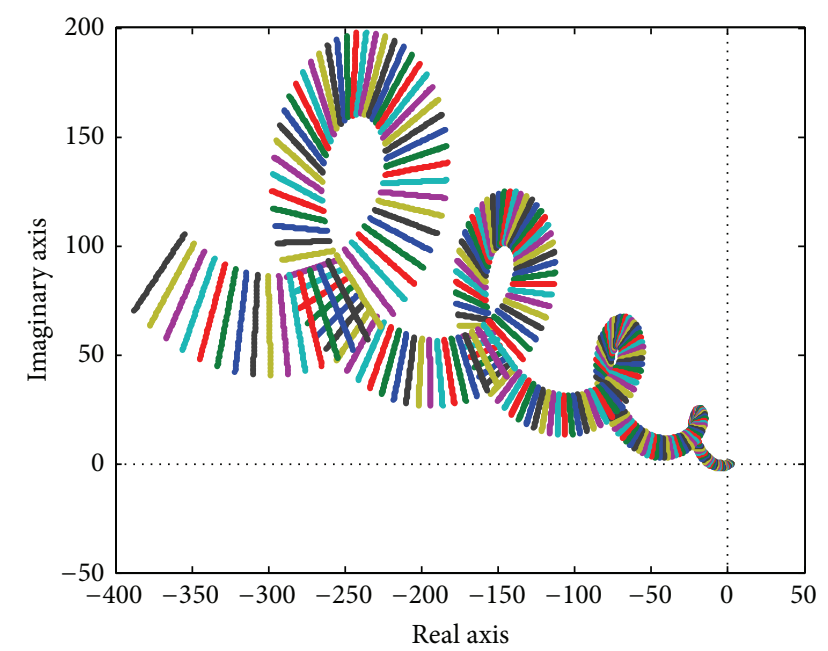

FIGURE 11: Value sets for controller (21) and plant (11) with parameters (12).

about fulfillment of all given preconditions, for example, the existence of at least one stable member of the analyzed family. If they are ignored, it can lead to the incorrect results. For example, consider again the same controlled plant with gain (12) and another PID controller with parameters:

$$
C(s)=K_{p}+\frac{K_{i}}{s}+K_{d} s=1.5+\frac{0.5}{s}+0.3 s
$$

The corresponding value sets (again for $\omega=0: 0.05: 15$ ) and the closer look at the origin are shown in Figures 13 and 14 , respectively.

Since the zero is obviously excluded from the value sets, it could (wrongly) indicate the robust stability of the family. However, the family does not have any stable member and so the zero exclusion condition is not fulfilled actually. In fact, all members of the family are unstable which is the reason why the stability border is not crossed at all and why the zero point

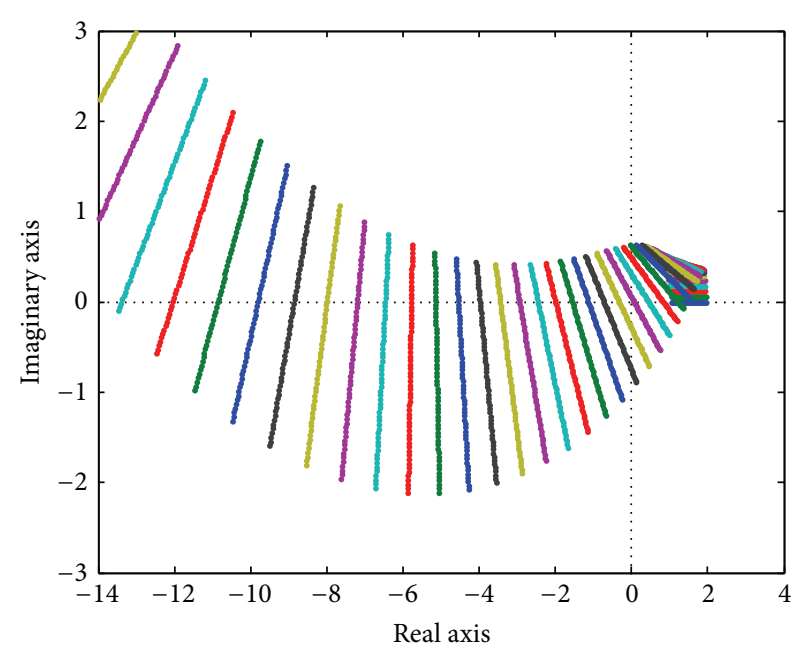

FIGURE 12: Value sets for controller (21) and plant (11) with parameters (12)-zoomed version.

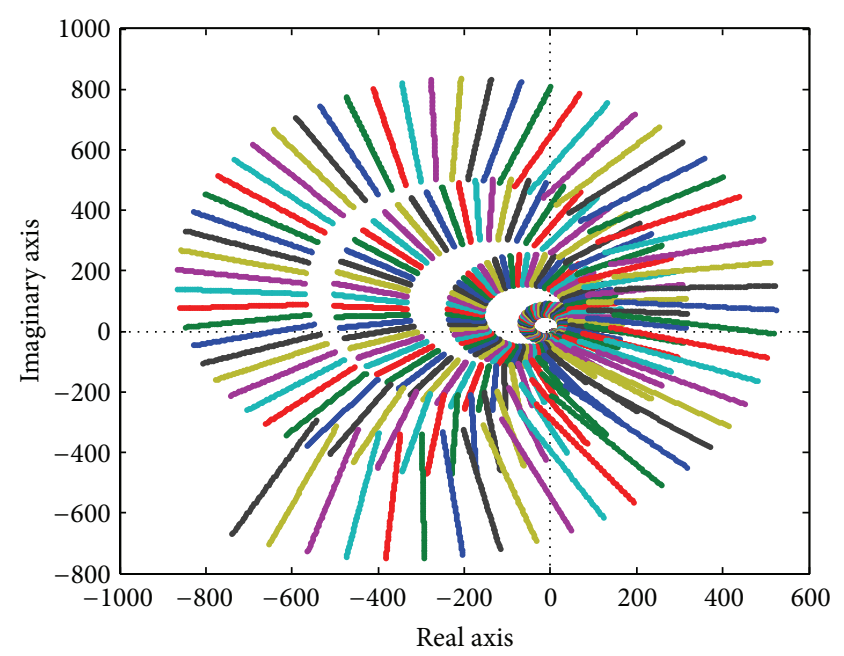

FIGURE 13: Value sets for controller (22) and plant (11) with parameters (12).

is not included. All in all, the family is not robustly stable and the assumed control loop would be unstable even for all possible values of $K$ from the prescribed interval.

\section{Example: Robust Stabilization of Main Irrigation Canals}

Whereas the previous examples from Section 4 have demonstrated primarily the basic utilization of the method and typical shapes of the value sets, the following example is based on real control of main irrigation canal pools [13,37].

Water is indispensable element for life and it is becoming the most valuable resource all over the world. Nowadays, irrigation is reported as the major water consuming activity [37] and thus control which will lead to more efficient water management in irrigation systems is required. 


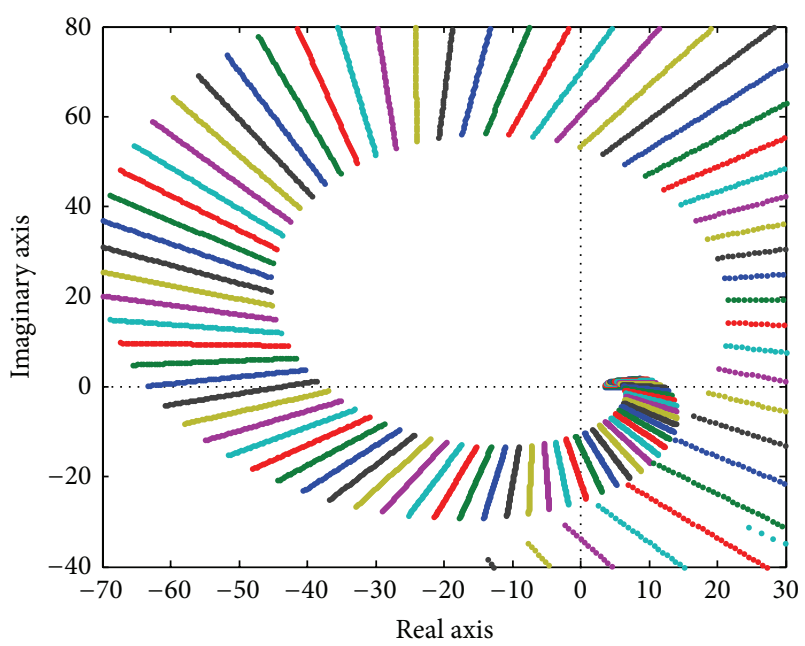

FIgURE 14: Value sets for controller (22) and plant (11) with parameters (12)—zoomed version.

In [37], the main irrigation canal pool was modelled as a second-order (integer order) transfer function with timedelay:

$$
G\left(s, K, T_{1}, \Theta\right)=\frac{K}{\left(T_{1} s+1\right)\left(T_{2} s+1\right)} e^{-\Theta s}
$$

where static gain $K$, time constant $T_{1}$, and time-delay term $\Theta$ are supposed to exhibit wide variations as a result of discharge through the upstream gate which varies in some operation range. The second time constant $T_{2}$ represents the motor and gate dynamics which is much faster than the dynamics of the canal pool and thus it is considered to be invariant. The nominal values of the uncertain parameters are $K_{0}=1.25$, $T_{10}=300[s]$, and $\Theta_{0}=600[s]$ and the fixed constant is $T_{2}=60[s]$.

Two controllers were designed in [37]. The first one is a classical PID controller:

$$
\begin{aligned}
C_{\mathrm{PID}}(s) & =K_{p}+\frac{K_{i}}{s}+K_{d} s \\
& =0.5511+\frac{0.0008}{s}+80.1334 s
\end{aligned}
$$

and the second one has the form of fractional order PI:

$$
C_{\mathrm{FPI}}(s)=\frac{K_{p} s+K_{i}}{s^{0.66}}=\frac{1.9964 s+0.0089}{s^{0.66}} .
$$

The maximal assumed variations of parameters from [37], that is, $0<K \leq 3.125,6 \leq T_{1} \leq 6000$, and $0<\Theta \leq$ 1800 , are really extreme and they lead to robustly unstable closed loop for both controllers. However, as the practical range of parameters should be much smaller, the intervals corresponding to $\pm 40 \%$ of the nominal values are supposed for the sake of robust stability analysis in this paper. That is,

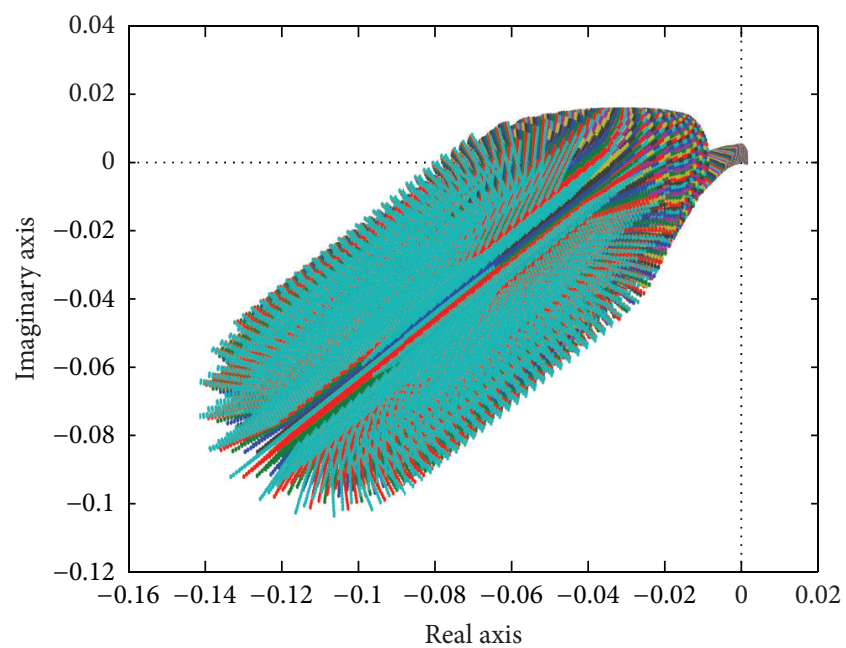

FIgURE 15: Value sets for controller (24) and plant (23) with parameters (26).

the considered uncertain parameters in transfer function (23) are as follows:

$$
\begin{aligned}
K & =[0.75,1.75] ; \\
T_{1} & =[180,420] ; \\
\Theta & =[360,840] .
\end{aligned}
$$

The corresponding families of closed-loop characteristic quasipolynomials are

$$
\begin{aligned}
p_{\mathrm{cl}-\mathrm{PID}}\left(s, K, T_{1}, \Theta\right)= & \left(T_{1} s+1\right)\left(T_{2} s+1\right) s \\
& +K e^{-\Theta s}\left(K_{d} s^{2}+K_{p} s+K_{i}\right)
\end{aligned}
$$

for PID controller (24) and

$$
\begin{aligned}
p_{\text {cl-FPI }}\left(s, K, T_{1}, \Theta\right)= & \left(T_{1} s+1\right)\left(T_{2} s+1\right) s^{0.66} \\
& +K e^{-\Theta s}\left(K_{p} s+K_{i}\right)
\end{aligned}
$$

for fractional order PI controller (25).

The sampling of frequency and parameters for the sake of the value sets visualization has been chosen as

$$
\begin{aligned}
\omega & =0: 0.0001: 0.015 \\
K & =0.75: 0.05: 1.75 \\
T_{1} & =180: 10: 420 \\
\Theta & =360: 20: 840 .
\end{aligned}
$$

The value sets for the family of quasipolynomials (27), that is, for PID controller (24) and family of systems (23) with parameters (26), are plotted in Figure 15. The zoomed complex plane origin is then shown in Figure 16. Since the family contains a stable member and the zero point is excluded from the value sets, the closed-loop control system is robustly stable. 


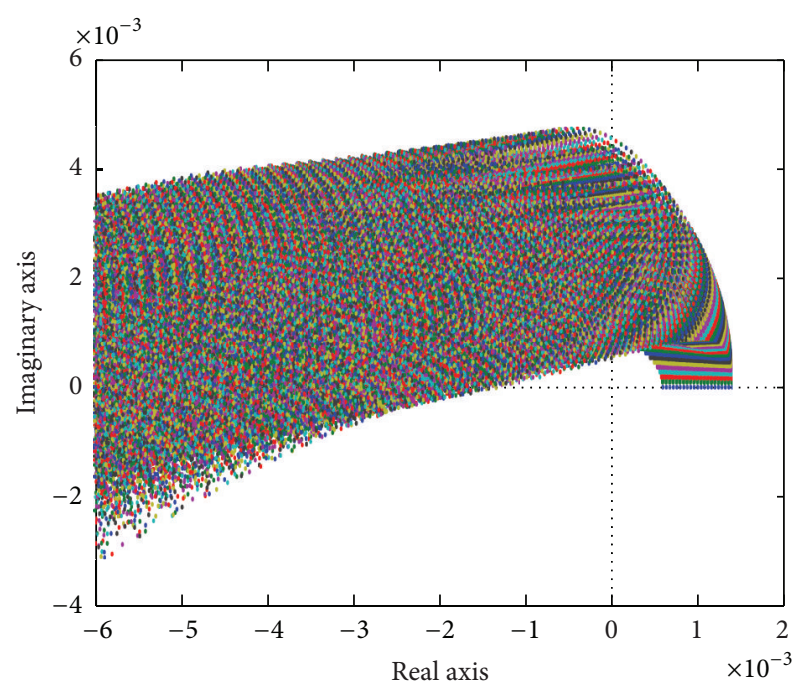

FIGURE 16: Value sets for controller (24) and plant (23) with parameters (26)-zoomed version.

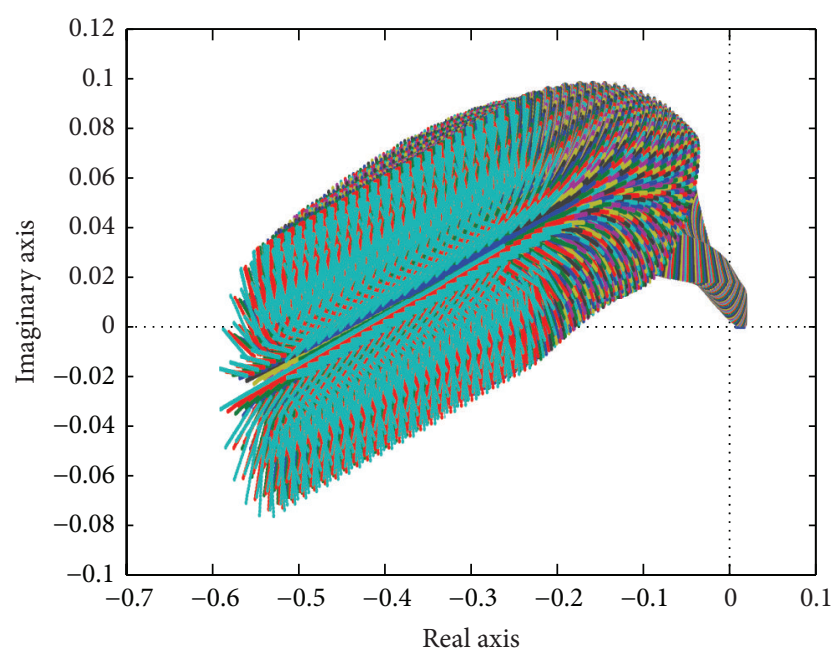

FIGURE 17: Value sets for controller (24) and plant (23) with parameters (26).

The value sets for the family of quasipolynomials (28), that is, for fractional order PI controller (25) and family of systems (23) with parameters (26), and the closer look are depicted in Figures 17 and 18, respectively. The final closedloop control system is robustly stable also in this case.

\section{Conclusion}

The main aim of the paper was to present a graphical approach to robust stability analysis and its application to fractional order time-delay feedback control loops consisting of a family of fractional order time-delay plants and either integer order or fractional order controller. The robust stability was verified through visualization of the value sets of a closed-loop characteristic quasipolynomial family and subsequent application of the zero exclusion condition

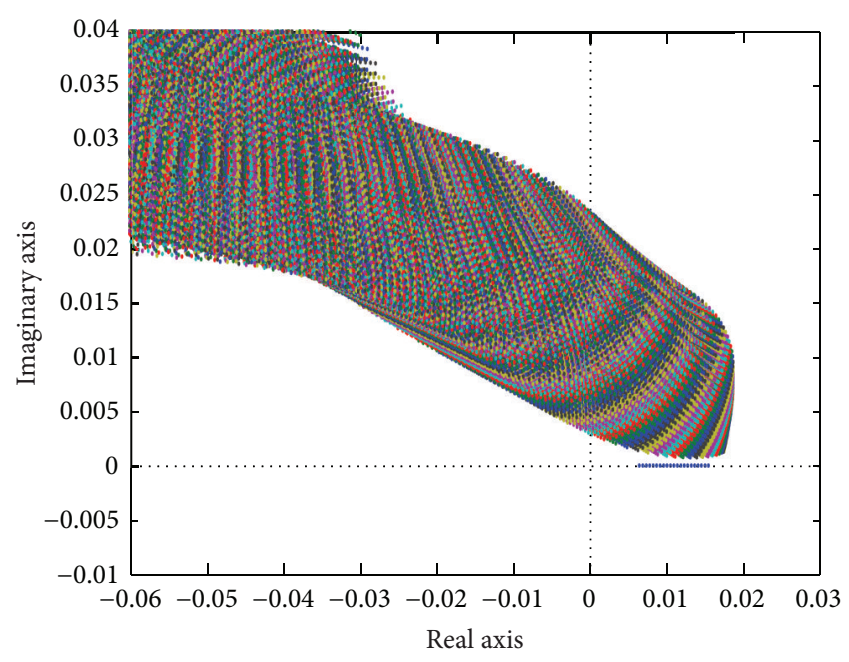

FIgURE 18: Value sets for controller (24) and plant (23) with parameters (26)-zoomed version.

for various combinations of uncertain parameters. Despite the fact that the presented computational examples from Section 4 combined the cases of fractional order plants with integer order controllers and the practically oriented example from Section 5 analyzed the integer order plant with integer order or fractional order controller, the combination of fractional order plant and fractional order controller is also effectively solvable by the presented graphical method.

\section{Conflict of Interests}

The authors declare that there is no conflict of interests regarding the publication of this paper.

\section{Acknowledgments}

The work was performed with financial support of research project NPU I no. MSMT-7778/2014 by the Ministry of Education of the Czech Republic and also by the European Regional Development Fund under the Project CEBIA-Tech no. CZ.1.05/2.1.00/03.0089.

\section{References}

[1] K. B. Oldham and J. Spanier, Fractional Calculus: Theory and Applications of Differentiation and Integration to Arbitrary Order, Academic Press, London, UK, 1974.

[2] K. S. Miller and B. Ross, An Introduction to the Fractional Calculus and Fractional Differential Equations, John Wiley \& Sons, New York, NY, USA, 1993.

[3] I. Podlubný, Fractional Differential Equations, Academic Press, San Diego, Calif, USA, 1999.

[4] Y. Q. Chen, I. Petráš, and D. Xue, "Fractional order controla tutorial," in Proceedings of the American Control Conference (ACC '09), pp. 1397-1411, St. Louis, Mo, USA, June 2009.

[5] I. Petráš, "Stability of fractional-order systems with rational orders: a survey," Fractional Calculus \& Applied Analysis, vol. 12, no. 3, pp. 269-298, 2009. 
[6] R. E. Gutiérrez, J. M. Rosário, and J. T. MacHado, "Fractional order calculus: basic concepts and engineering applications," Mathematical Problems in Engineering, vol. 2010, Article ID 375858, 19 pages, 2010.

[7] R. Matušů, "Application of fractional order calculus to control theory," International Journal of Mathematical Models and Methods in Applied Sciences, vol. 5, no. 7, pp. 1162-1169, 2011.

[8] M. Axtell and M. E. Bise, "Fractional calculus applications in control systems," in Proceedings of the IEEE National Aerospace and Electronics Conference (NAECON '90), pp. 563-566, Dayton, Ohio, USA, May 1990.

[9] S. E. Hamamci, "Stabilization using fractional-order PI and PID controllers," Nonlinear Dynamics, vol. 51, no. 1-2, pp. 329-343, 2008.

[10] S. E. Hamamci and M. Koksal, "Calculation of all stabilizing fractional-order PD controllers for integrating time delay systems," Computers and Mathematics with Applications, vol. 59, no. 5, pp. 1621-1629, 2010.

[11] I. Podlubný, "Fractional-order systems and -controllers," IEEE Transactions on Automatic Control, vol. 44, no. 1, pp. 208-214, 1999.

[12] C. Yeroğlu and N. Tan, "Classical controller design techniques for fractional order case," ISA Transactions, vol. 50, no. 3, pp. 461-472, 2011.

[13] A. Mesbahi and M. Haeri, "Robust non-fragile fractional order PID controller for linear time invariant fractional delay systems," Journal of Process Control, vol. 24, no. 9, pp. 1489-1494, 2014.

[14] Z. Gao, M. Yan, and J. Wei, "Robust stabilizing regions of fractional-order $\mathrm{PD} \mu$ controllers of time-delay fractional-order systems," Journal of Process Control, vol. 24, no. 1, pp. 37-47, 2014.

[15] C. Hwang and Y.-C. Cheng, "A numerical algorithm for stability testing of fractional delay systems," Automatica, vol. 42, no. 5, pp. 825-831, 2006.

[16] C. Bonnet and J. R. Partington, "Analysis of fractional delay systems of retarded and neutral type," Automatica, vol. 38, no. 8, pp. 1133-1138, 2002.

[17] C. Bonnet and J. R. Partington, "Stabilization of some fractional delay systems of neutral type," Automatica, vol. 43, no. 12, pp. 2047-2053, 2007

[18] N. Tan, Ö. Faruk Özgüven, and M. Mine Özyetkin, "Robust stability analysis of fractional order interval polynomials," ISA Transactions, vol. 48, no. 2, pp. 166-172, 2009.

[19] B. Şenol and C. Yeroğlu, "Robust stability analysis of fractional order uncertain polynomials," in Proceedings of the 5th IFAC Workshop on Fractional Differentiation and Its Applications, Nanjing, China, 2012.

[20] B. Şenol and C. Yeroğlu, "Computation of the value set of fractional order uncertain polynomials: a 2q convex parpolygonal approach," in Proceedings of the IEEE International Conference on Control Applications (CCA '12), pp. 686-691, Dubrovnik, Croatia, October 2012.

[21] C. Yeroğlu and B. Şenol, "Investigation of robust stability of fractional order multilinear affine systems: 2q-convex parpolygon approach," Systems \& Control Letters, vol. 62, no. 10, pp. 845$855,2013$.

[22] B. Senol, A. Ateş, B. Baykant Alagoz, and C. Yeroğlu, "A numerical investigation for robust stability of fractional-order uncertain systems," ISA Transactions, vol. 53, no. 2, pp. 189-198, 2014.
[23] K. A. Moornani and M. Haeri, "Robust stability testing function and Kharitonov-like theorem for fractional order interval systems," IET Control Theory \& Applications, vol. 4, no. 10, pp. 2097-2108, 2010.

[24] J.-G. Lu and Y. Chen, "Stability and stabilization of fractionalorder linear systems with convex polytopic uncertainties," Fractional Calculus \& Applied Analysis, vol. 16, no. 1, pp. 142$157,2013$.

[25] B. Senol and C. Yeroğlu, "Frequency boundary of fractional order systems with nonlinear uncertainties," Journal of the Franklin Institute, vol. 350, no. 7, pp. 1908-1925, 2013.

[26] C. Li and J. Wang, "Robust stability and stabilization of fractional order interval systems with coupling relationships: the $0<\alpha<1$ case," Journal of the Franklin Institute, vol. 349, no. 7, pp. 2406-2419, 2012.

[27] Z. Liao, C. Peng, W. Li, and Y. Wang, "Robust stability analysis for a class of fractional order systems with uncertain parameters," Journal of the Franklin Institute, vol. 348, no. 6, pp. 11011113, 2011.

[28] S. Zheng, X. Tang, and B. Song, "A graphical tuning method of fractional order proportional integral derivative controllers for interval fractional order plant," Journal of Process Control, vol. 24, no. 11, pp. 1691-1709, 2014.

[29] R. Matušů and R. Prokop, "Graphical analysis of robust stability for fractional order time-delay systems and integer order PID controllers," in Proceedings of the 18th International Conference on Systems, Santorini, Greece, 2014.

[30] B. R. Barmish, New Tools for Robustness of Linear Systems, Macmillan, New York, NY, USA, 1994.

[31] R. Matušů and R. Prokop, "Graphical analysis of robust stability for systems with parametric uncertainty: an overview," Transactions of the Institute of Measurement and Control, vol. 33, no. 2, pp. 274-290, 2011.

[32] R. Matušů and R. Prokop, "Robust stability analysis for systems with real parametric uncertainty: implementation of graphical tests in Matlab," International Journal of Circuits, Systems and Signal Processing, vol. 7, no. 1, pp. 26-33, 2013.

[33] A. Tepljakov, "FOMCON: Fractional-Order Modeling and Control," 2013, http://fomcon.net/.

[34] A. Tepljakov, E. Petlenkov, and J. Belikov, "FOMCON: fractional-order modeling and control toolbox for MATLAB," in Proceedings of the 18th International Conference "Mixed Design of Integrated Circuits and Systems", Gliwice, Poland, 2011.

[35] A. Oustaloup, F. Levron, B. Mathieu, and F. M. Nanot, "Frequency-band complex noninteger differentiator: characterization and synthesis," IEEE Transactions on Circuits and Systems-I: Fundamental Theory and Applications, vol. 47, no. 1, pp. 25-39, 2000.

[36] W. Krajewski and U. Viaro, "A method for the integer-order approximation of fractional-order systems," Journal of the Franklin Institute, vol. 351, no. 1, pp. 555-564, 2014.

[37] V. Feliu-Batlle, R. R. Pérez, and L. S. Rodríguez, "Fractional robust control of main irrigation canals with variable dynamic parameters," Control Engineering Practice, vol. 15, no. 6, pp. 673686, 2007. 


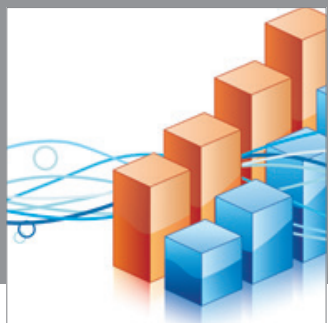

Advances in

Operations Research

mansans

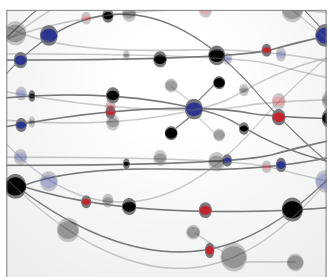

The Scientific World Journal
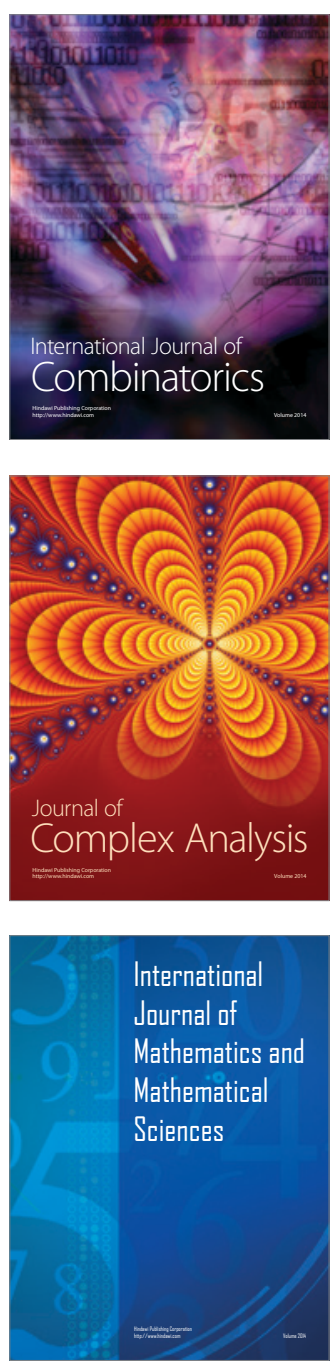
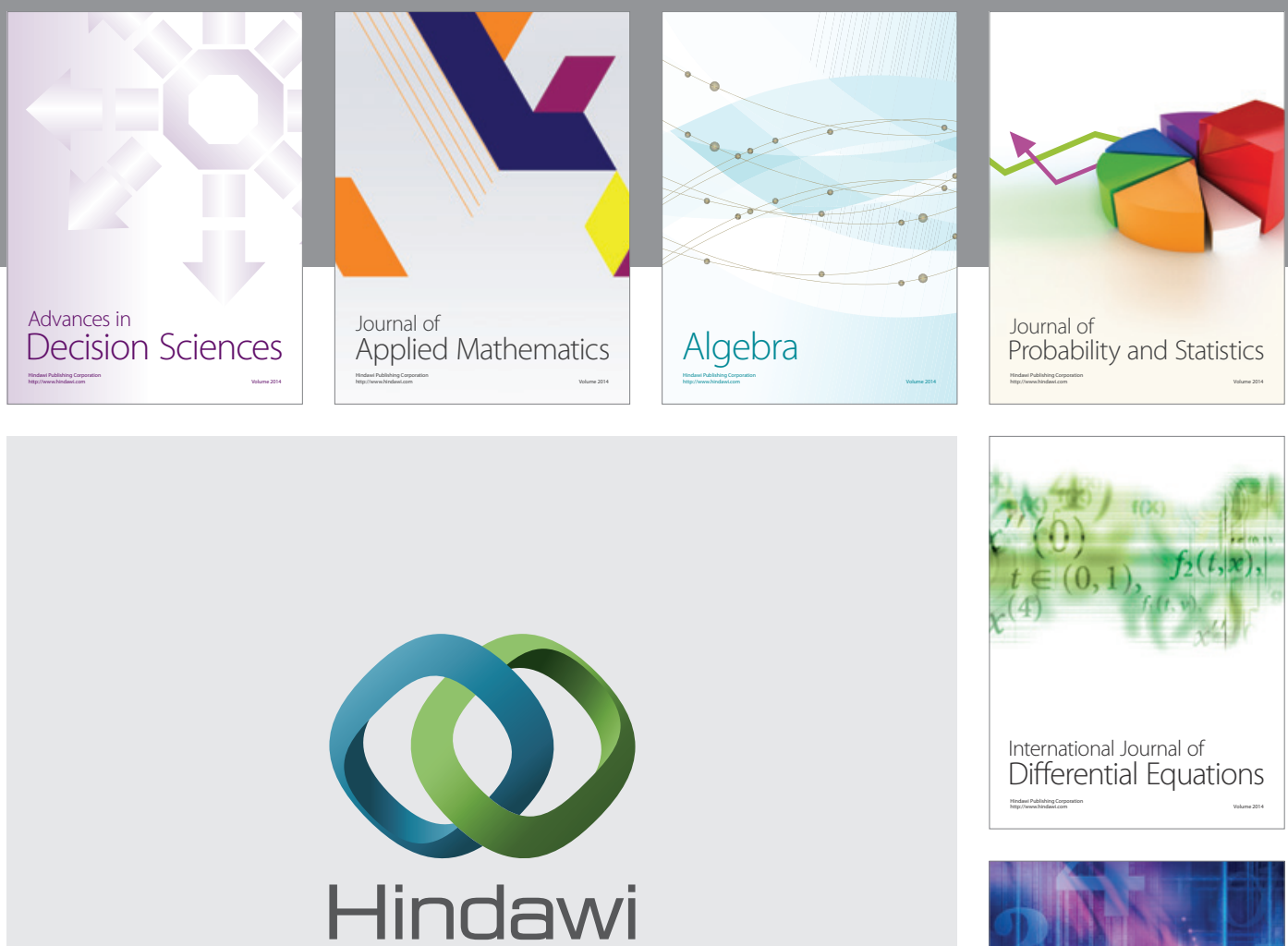

Submit your manuscripts at http://www.hindawi.com
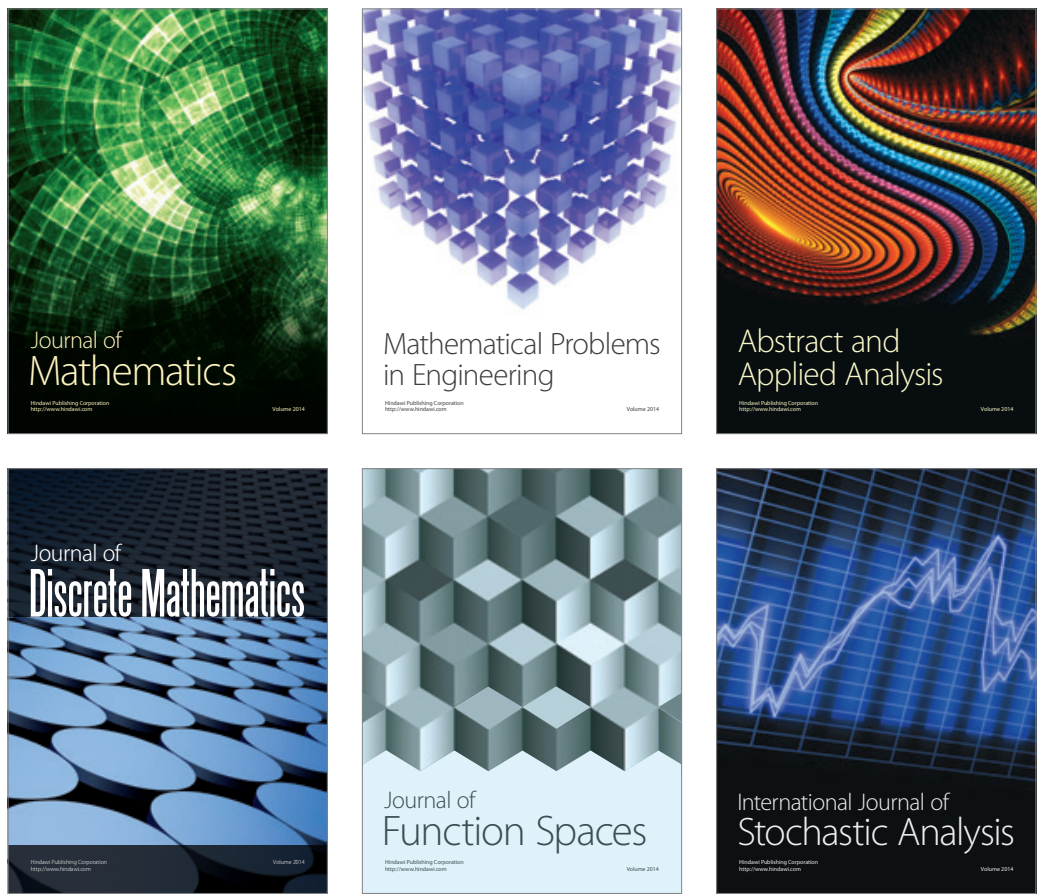

Journal of

Function Spaces

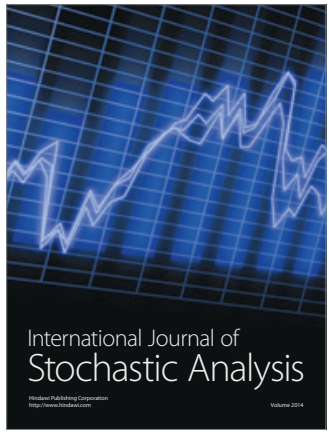

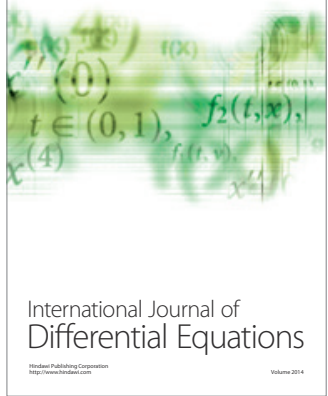
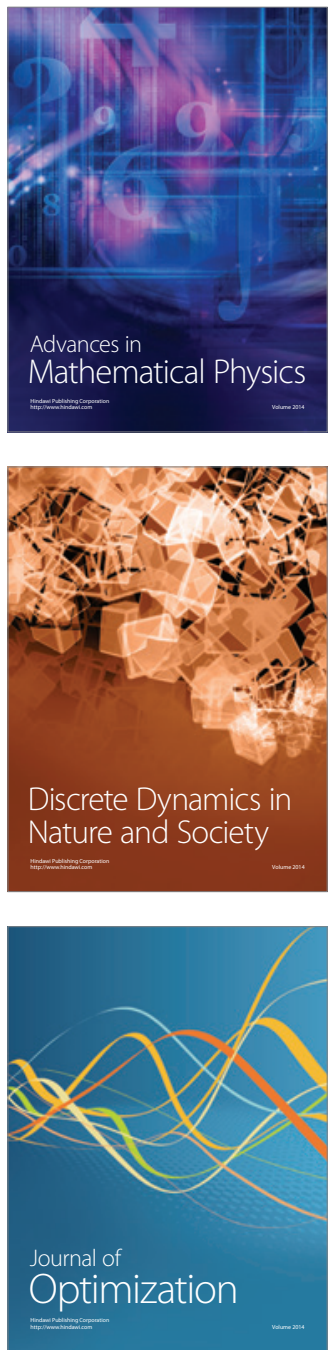